ISSN 1392-3196 / e-ISSN 2335-8947

Zemdirbyste-Agriculture, vol. 100, No. 4 (2013), p. 433-440

DOI 10.13080/z-a.2013.100.055

\title{
Simple sequence repeat markers associated with agro-morphological traits in chickpea (Cicer arietinum $\mathrm{L}$.)
}

\author{
Ali SAEED ${ }^{1}$, Reza DARVISHZADEH ${ }^{2,3}$, Ashkan BASIRNIA $^{2}$ \\ ${ }^{1}$ West Azerbaijan Agricultural and Natural Resources Research Centre \\ Urmia, Iran \\ ${ }^{2}$ Department of Plant Breeding and Biotechnology, Urmia University \\ Urmia, Iran \\ E-mail: r.darvishzadeh@urmia.ac.ir \\ ${ }^{3}$ Institute of Biotechnology, Urmia University \\ Urmia, Iran
}

\begin{abstract}
Chickpea (Cicer arietinum L.), a cool season grain legume, serves as an important cheap source of protein and energy in developing countries, and plays an important role in the enrichment of soil fertility. In order to identify simple sequence repeat (SSR) markers associated with agro-morphological traits in chickpea, 44 genotypes comprising cultigen, landraces, internationally developed improved lines and wild relatives, were phenotyped for several agro-morphological traits in a randomized complete block design with three replications at Urmia Rainfed Research Station. High genetic variability was observed among the chickpea genotypes for the studied agro-morphological traits. In molecular experiment, genetic variability among genotypes was assessed by using SSR markers. One hundred alleles were generated at 16 SSR loci, with a mean number of alleles per locus of 6.25. Using general linear model (GLM) and applying multiple testing corrections, 10 SSR loci associated with the genes controlling the studied agro-morphological traits were identified. Some identified markers were associated with more than one trait. The identified markers could be of great interest in marker assisted selection (MAS) in chickpea breeding programmes.
\end{abstract}

Key words: association mapping, Cicer arietinum, false positive, genetic diversity, molecular markers.

\section{Introduction}

Chickpea as a third major cool season grain legume in the world has an important role in poor people's diet. The cultivated chickpea (Cicer arietinum L.) is grown on 11 million hectares with 9.77 million ton production (http://faostat.fao.org). The world average yield $\left(0.88 \mathrm{tha}^{-1}\right)$ is far below potential seed yield of about $5 \mathrm{t} \mathrm{ha}^{-1}$ (Ahmad et al., 2005) due to complex field environment with its heterogeneous conditions and global climatic changes (Mittler, Blumwald, 2010), lack of well adapted cultivars for autumn or winter sowing, poor farm management and susceptibility to several biotic and abiotic stresses.

Ascochyta blight, caused by the fungus Ascochyta rabiei, is a damaging disease of chickpeas in Iran (Kanouni et al., 2010). The fungus has the potential to attack all above ground parts of the plant which may cause $100 \%$ yield loss (Rajesh, Muehlbauer, 2008) and is most prevalent in the areas where cool, cloudy and humid weather occurs during the crop season (Kanouni et al., 2010). Developing ascochyta blight resistant varieties is the most economically and environmentally sound means of controlling the disease.

Cold stress generally affects chickpea growth and development. Low temperature has been considered as a primary limiting factor in most regions and has been therefore the focus of most research works. Chickpea is the least cold tolerant crop among the cool-season food legumes. The severity of cold stress depends on the rate of cooling, minimal temperature and its duration. There is significant variability for cold tolerance in chickpea germplasms at early growth stage (Saeed et al., 2010).

Molecular breeding has revolutionized conventional breeding techniques in all areas within the last twenty years. It has played a significant role in chickpea breeding programs by integrating marker assisted selection (MAS) within empirical selection (Chaturvedi, Nadarajan, 2010). As a prerequisite, identifying tightly linked markers is important for the successful deployment of molecular marker technology within a breeding programme. Molecular markers linked with quantitative trait loci (QTL)/major genes for traits of interest are being routinely identified in many crops by utilizing genetic linkage map developed on the second filial generation (F2) or recombinant inbred lines (RILs) populations. Genetic linkage maps have been developed for chickpea in different research works (Jain, Chattopadhyay, 2010; Gaur et al., 2011), and QTLs 
controlling different traits such as double podding and other morphological traits (Gaur et al., 2011), as well as drought tolerance (Jain, Chattopadhyay, 2010) have been identified. Identification of markers associated with important traits in a group of genotypes through association mapping offers an alternative means, as has been used in several plants species (Vijayan et al., 2005; Roy et al., 2006; Darvishzadeh et al., 2008). Roy et al. (2006) by using the phenotypic data of 55 elite wheat genotypes for 14 different agronomic traits and molecular data produced by 20 simple sequence repeat (SSR), 2 selectively amplified microsatellite polymorphic loci (SAMPL) and 8 amplified fragment length polymorphism (AFLP) primer combinations (in total 519 deoxyribonucleic acid (DNA)-based molecular markers), reported some molecular markers associated with traits via simple or multiple linear regression analysis. In their study, a total of 131 SSR, 43 SAMPL and 166 AFLP markers showed significant associations with at least one of the 14 studied traits. Darvishzadeh et al. (2008) by using multiple linear regression analysis identified putative AFLP markers associated with partial resistance to phoma black stem in 60 sunflower mutant lines. Vijayan et al. (2005) genotyped 44 mulberry genotypes with inter-simple sequence repeat (ISSR) markers and identified putative markers associated with leaf yield attributing traits. The potential of association mapping to identify and characterize loci/genes associated with different complex traits is highly affected by admixture of populations (Zhang et al., 2012). Therefore, knowledge on the population structure is a prerequisite in association mapping and can be used to avoid identifying false positive correlations between markers and traits (Pritchard et al., 2000; Pritchard, Donnelly, 2001).
The objectives of the present study were to characterize the population structure within chickpea genotypes and to identify SSR markers associated with agro-morphological traits in chickpea using association mapping by general linear model (GLM) procedure.

\section{Materials and methods}

Plant material and phenotypic data. A set of 44 genotypes of chickpea out of 225 genotypes (Saeed et al., 2010) comprising cultigen, landraces, internationally developed improved lines and wild relatives (Table 1), were planted in a randomized complete block design with three replications at Urmia Rainfed Research Station. The accessions were planted in two rows with 4 meters length and 30 centimetres width on 12 October, 2009. Traits including number of stems (NS), plant height $(\mathrm{PH})$, cold tolerance $(\mathrm{CT})$, days from the first effective raining after sowing to $50 \%$ flowering (DF), days from the first effective raining after sowing to $90 \%$ maturity (DM), susceptibility to ascochyta blight disease (SABD), 100 -seed weight (100 SW) and seed yield (YLD) were recorded (Table 1). For the visual screening of cold tolerance, we used a scale of $1-9$, where 1 - tolerant, no visible symptoms of damage; 3 - tolerant, slight foliar damage (11-20\% leaflets show withering) and up to $20 \%$ branches show withering and drying, no plant killing; 5 - intermediate, $41-60 \%$ leaflets and $21-40 \%$ branches show withering and drying, up to 5\% plant killing; 7 susceptible, $81-99 \%$ leaflets and 61-90\% branches show withering and drying, 26-50\% plant killing; and 9- highly susceptible, $100 \%$ plant killing (International Center for Agricultural Research in the Dry Areas (ICARDA) manual, 2008). Pathological tests were performed by using the method described by Kanouni et al. (2010).

Table 1. Mean and standard error values of studied agro-morphological characteristics in 44 genotypes of chickpea comprising cultigen, landraces, internationally developed improved lines and wild relatives, evaluated in a randomized complete block design with three replications at Urmia Rainfed Research Station

\begin{tabular}{|c|c|c|c|c|c|c|c|c|c|c|c|c|c|c|c|c|c|c|c|c|c|}
\hline \multirow{2}{*}{ No. } & \multirow{2}{*}{ Genotype } & \multirow{2}{*}{ Origin } & \multirow{2}{*}{ Pedigree } & \multirow{2}{*}{ Type } & \multirow{2}{*}{$\begin{array}{c}\text { Sub } \\
\text { group }^{\mathrm{b}}\end{array}$} & \multicolumn{2}{|c|}{ NS } & \multicolumn{2}{|c|}{$\begin{array}{c}\text { DF } \\
\text { (days) }\end{array}$} & \multicolumn{2}{|c|}{$\begin{array}{c}\mathrm{DM} \\
\text { (days) }\end{array}$} & \multicolumn{2}{|c|}{ SABD } & \multicolumn{2}{|c|}{$\mathrm{CT}$} & \multicolumn{2}{|c|}{$\begin{array}{l}\mathrm{PH} \\
\mathrm{cm}\end{array}$} & \multicolumn{2}{|c|}{$\begin{array}{c}100 \mathrm{SW} \\
\mathrm{g}\end{array}$} & \multicolumn{2}{|c|}{$\begin{array}{c}\text { YLD } \\
\mathrm{kg} \mathrm{ha}^{-1}\end{array}$} \\
\hline & & & & & & $\bar{X}$ & $\mathrm{SE}$ & $\bar{X}$ & $\mathrm{SE}$ & $\bar{X}$ & SE & $\bar{X}$ & $\mathrm{SE}$ & $\bar{X}$ & $\mathrm{SE}$ & $\bar{X}$ & SE & $\bar{X}$ & $\mathrm{SE}$ & $\bar{X}$ & SE \\
\hline 1 & ILC533 & Egypt & not traced & Kabuli & mixed & 2 & 1.00 & 185 & 0.33 & 225 & 1.53 & 7 & 1.33 & 9 & 0.59 & 52 & 6.11 & 16.1 & 4.38 & 145.0 & 37.48 \\
\hline 2 & ILC 3279 & $\begin{array}{l}\text { Former } \\
\text { USSR }\end{array}$ & landrace & Kabuli & red & 2 & 0.00 & 184 & 0.67 & 226 & 1.53 & 2 & 0.33 & 8 & 0.00 & 51 & 0.882 & 28.2 & 0.09 & 728.0 & 141.91 \\
\hline 3 & ILC 8262 & Spain & $\begin{array}{l}\text { selection from } \\
\text { ILC } 3470\end{array}$ & Kabuli & red & 4 & 0.67 & 182 & 0.67 & 220 & 3.33 & 2 & 0.33 & 3 & 0.00 & 51 & 2.192 & 26.4 & 0.41 & 4190.4 & 1322.33 \\
\hline 4 & ILC 8617 & ICARDA & $\begin{array}{l}\text { ILC482 } \\
\text { (mutation) }\end{array}$ & Kabuli & mixed & 3 & 0.33 & 180 & 0.33 & 225 & 1.20 & 2 & 0.33 & 3 & 0.00 & 44 & 2.192 & 27.3 & 0.49 & 842.0 & 186.25 \\
\hline 5 & ILWC 81 & Turkey & $\begin{array}{l}\text { C. reticulatum } \\
\text { IG }\end{array}$ & wild & red & 5 & 0.33 & 174 & 4.00 & 220 & 2.96 & 2 & 2.19 & 2 & 0.33 & 46 & 8.41 & 15.0 & 5.72 & 266.0 & 62.80 \\
\hline 6 & ILWC 106 & Turkey & $\begin{array}{l}\text { C. echinusper- } \\
\text { mum IG }\end{array}$ & wild & red & 6 & 0.88 & 179 & 2.52 & 220 & 2.96 & 2 & 0.33 & 2 & 0.33 & 31 & 1.00 & 19.5 & 1.96 & 251.8 & 43.44 \\
\hline 7 & ILWC 139 & Turkey & $\begin{array}{l}\text { C. judaicum } \\
\text { IG }\end{array}$ & wild & mixed & 7 & 1.00 & 172 & 4.67 & 220 & 2.96 & 2 & 0.67 & 2 & 0.33 & 18 & 4.48 & 18.2 & 2.01 & 197.6 & 33.72 \\
\hline 8 & ILWC 181 & Turkey & $\begin{array}{l}\text { C. reticulatum } \\
\text { IG }\end{array}$ & wild & mixed & 3 & 0.33 & 179 & 0.33 & 220 & 3.33 & 2 & 0.58 & 2 & 0.33 & 23 & 0.00 & 15.2 & 3.66 & 31.8 & 20.88 \\
\hline 9 & ILWC 235 & Turkey & $\begin{array}{l}\text { C. echinusper- } \\
\text { mum IG }\end{array}$ & wild & red & 5 & 0.33 & 176 & 3.93 & 220 & 3.18 & 2 & 0.88 & 2 & 0.33 & 16 & 5.78 & 19.5 & 1.53 & 583.0 & 160.32 \\
\hline 10 & Shahindezh & Iran & landrace & Desi & red & 3 & 0.00 & 176 & 0.58 & 221 & 0.88 & 3 & 0.00 & 8 & 0.00 & 38 & 0.33 & 18.2 & 0.64 & 622.0 & 148.35 \\
\hline 11 & $\begin{array}{l}\text { Germezi } \\
\text { Nukhud }\end{array}$ & Iran & landrace & Desi & red & 3 & 0.00 & 175 & 0.33 & 220 & 1.15 & 3 & 0.00 & 8 & 0.00 & 42 & 1.33 & 19.5 & 0.99 & 590.2 & 121.09 \\
\hline 12 & Mahabad & Iran & landrace & Desi & red & 3 & 0.00 & 176 & 1.15 & 220 & 1.20 & 3 & 0.00 & 4 & 0.00 & 44 & 2.40 & 18.3 & 0.72 & 691.2 & 170.64 \\
\hline 13 & Kaka & Iran & not traced & Desi & red & 4 & 0.33 & 186 & 3.33 & 220 & 1.67 & 2 & 0.67 & 4 & 0.33 & 64 & 8.84 & 12.0 & 1.58 & 110.8 & 29.69 \\
\hline
\end{tabular}


Table 1 continued

\begin{tabular}{|c|c|c|c|c|c|c|c|c|c|c|c|c|c|c|c|c|c|c|c|c|c|}
\hline \multirow{2}{*}{ No. } & \multirow{2}{*}{ Genotype } & \multirow{2}{*}{ Origin } & \multirow{2}{*}{ Pedigree } & \multirow{2}{*}{ Type } & \multirow{2}{*}{$\begin{array}{c}\text { Sub } \\
\text { group }^{b}\end{array}$} & \multicolumn{2}{|c|}{ NS } & \multicolumn{2}{|c|}{$\begin{array}{c}\mathrm{DF} \\
\text { (days) }\end{array}$} & \multicolumn{2}{|c|}{$\begin{array}{c}\mathrm{DM} \\
\text { (days) }\end{array}$} & \multicolumn{2}{|c|}{ SABD } & \multicolumn{2}{|c|}{$\mathrm{CT}$} & \multicolumn{2}{|c|}{$\begin{array}{l}\mathrm{PH} \\
\mathrm{cm}\end{array}$} & \multicolumn{2}{|c|}{$\begin{array}{c}100 \mathrm{SW} \\
\mathrm{g}\end{array}$} & \multicolumn{2}{|c|}{$\begin{array}{c}\text { YLD } \\
\mathrm{kg} \mathrm{ha}^{-1}\end{array}$} \\
\hline & & & & & & $\bar{X}$ & $\mathrm{SE}$ & $\bar{X}$ & $\mathrm{SE}$ & $\bar{X}$ & $\mathrm{SE}$ & $\bar{X}$ & SE & $\bar{X}$ & $\mathrm{SE}$ & $\bar{X}$ & SE & $\bar{X}$ & SE & $\bar{X}$ & SE \\
\hline 14 & Piruz & Iran & not traced & Desi & green & 4 & 0.33 & 173 & 0.88 & 220 & 1.86 & 2 & 0.67 & 3 & 0.33 & 37 & 1.53 & 17.4 & 4.86 & 514.8 & 99.87 \\
\hline 15 & Ghazvin & Iran & landrace & Kabuli & green & 4 & 0.33 & 172 & 0.88 & 222 & 0.58 & 2 & 1.00 & 6 & 0.00 & 56 & 4.84 & 35.8 & 0.29 & 2411.0 & 693.61 \\
\hline 16 & $\begin{array}{l}\text { X 96TH3 } \\
\text { K3 }\end{array}$ & ICARDA & $\begin{array}{l}\text { FLIP } 91-150 \mathrm{C} \times \\
\text { FLIP } 93-93 \mathrm{C}\end{array}$ & Kabuli & green & 3 & 0.00 & 181 & 2.91 & 221 & 16.04 & 2 & 0.67 & 5 & 0.00 & 67 & 6.57 & 32.7 & 0.46 & 2830.4 & 852.97 \\
\hline 17 & $\begin{array}{l}\text { X 95TH5 } \\
\text { K10 }\end{array}$ & ICARDA & $\begin{array}{l}\text { FLIP 91-149C } \times \\
\text { FLIP 93-194C }\end{array}$ & Kabuli & mixed & 3 & 0.33 & 176 & 3.38 & 220 & 2.67 & 2 & 0.33 & 6 & 0.00 & 60 & 1.73 & 31.9 & 1.62 & 2970.4 & 696.41 \\
\hline 18 & $\begin{array}{l}\text { Sel } 93 \mathrm{TH} 2 \\
4416\end{array}$ & ICARDA & $\begin{array}{l}\text { ILC482 } \times \\
\text { NEWC } 36\end{array}$ & Desi & mixed & 3 & 0.67 & 169 & 4.37 & 220 & 1.67 & 6 & 1.45 & 5 & 0.00 & 39 & 6.74 & 25.5 & 2.08 & 1975.0 & 461.79 \\
\hline 19 & $\begin{array}{c}\text { Sel 93TH2 } \\
4477\end{array}$ & ICARDA & $\begin{array}{l}\text { ILC } 3470 \times \\
\text { ILC } 8617\end{array}$ & Desi & pink & 4 & 0.33 & 170 & 4.37 & 220 & 2.00 & 6 & 1.20 & 4 & 0.00 & 53 & 5.17 & 27.0 & 0.89 & 1784.4 & 413.45 \\
\hline 20 & $\begin{array}{l}\text { Sel 95TH1 } \\
716\end{array}$ & ICARDA & $\begin{array}{l}\text { ILC482 } \times \\
\text { ILWC182 }\end{array}$ & Desi & pink & 3 & 0.33 & 170 & 3.51 & 220 & 1.76 & 5 & 0.67 & 3 & 0.00 & 48 & 6.00 & 27.3 & 0.78 & 3123.4 & 745.54 \\
\hline 21 & $\begin{array}{l}\text { Sel 95TH1 } \\
744\end{array}$ & ICARDA & $\begin{array}{l}\text { ILC482 } \times \\
\text { NEWC } 36\end{array}$ & Desi & pink & 4 & 0.67 & 169 & 3.84 & 220 & 1.53 & 8 & 1.20 & 5 & 0.33 & 31 & 2.67 & 28.2 & 1.05 & 528.2 & 77.75 \\
\hline 22 & $\begin{array}{l}\text { Sel 95TH1 } \\
745\end{array}$ & ICARDA & $\begin{array}{l}\text { ILC482 } \times \\
\text { NEWC } 36\end{array}$ & Desi & pink & 4 & 0.33 & 168 & 4.37 & 220 & 1.76 & 8 & 1.15 & 3 & 0.33 & 39 & 4.41 & 27.3 & 1.79 & 615.4 & 167.21 \\
\hline 23 & $\begin{array}{l}\text { Sel 96TH1 } \\
1403\end{array}$ & ICARDA & $\begin{array}{l}\text { ILC482 } \times \\
\text { NEWC } 36\end{array}$ & Desi & blue & 4 & 0.58 & 168 & 4.37 & 220 & 1.86 & 8 & 1.76 & 2 & 0.33 & 30 & 6.49 & 26.4 & 0.91 & 314.0 & 20.11 \\
\hline 24 & $\begin{array}{l}\text { Sel 96TH1 } \\
1404\end{array}$ & ICARDA & $\begin{array}{l}\text { ILC482 } \times \\
\text { NEWC } 36\end{array}$ & Desi & blue & 4 & 0.33 & 168 & 4.33 & 220 & 1.67 & 8 & 1.20 & 3 & 0.33 & 36 & 2.89 & 26.6 & 0.36 & 302.2 & 57.04 \\
\hline 25 & $\begin{array}{l}\text { Sel 96TH1 } \\
1406\end{array}$ & ICARDA & $\begin{array}{l}\text { ILC482 } \times \\
\text { NEWC } 36\end{array}$ & Desi & blue & 4 & 0.00 & 170 & 5.03 & 220 & 2.40 & 8 & 1.76 & 3 & 0.33 & 35 & 5.03 & 28.7 & 1.45 & 802.4 & 193.57 \\
\hline 26 & $\begin{array}{c}\text { Sel 96TH1 } \\
1439\end{array}$ & ICARDA & $\begin{array}{c}\text { ILC482 } \times \text { ILWC } \\
182\end{array}$ & Desi & mixed & 4 & 0.00 & 168 & 4.00 & 225 & 1.53 & 4 & 0.58 & 2 & 0.33 & 52 & 6.77 & 27.3 & 0.12 & 1932.8 & 414.53 \\
\hline 27 & $\begin{array}{l}\text { Sel 96TH1 } \\
1484\end{array}$ & ICARDA & $\begin{array}{l}\text { ILC482 } \times \\
\text { NEWC } 36\end{array}$ & Desi & yellow & 4 & 0.33 & 170 & 7.02 & 220 & 3.51 & 8 & 1.45 & 3 & 0.33 & 30 & 5.03 & 26.5 & 2.38 & 663.8 & 176.68 \\
\hline 28 & $\begin{array}{l}\text { Sel 96TH1 } \\
1485\end{array}$ & ICARDA & $\begin{array}{l}\text { ILC482 } \times \\
\text { NEWC } 36\end{array}$ & Desi & yellow & 4 & 0.33 & 172 & 5.03 & 220 & 2.96 & 7 & 0.67 & 2 & 0.33 & 34 & 2.52 & 26.2 & 2.91 & 945.0 & 218.51 \\
\hline 29 & $\begin{array}{l}\text { Sel 96TH1 } \\
1488\end{array}$ & ICARDA & $\begin{array}{l}\text { ILC482 } \times \\
\text { NEWC } 36\end{array}$ & Desi & yellow & 3 & 0.33 & 172 & 6.77 & 220 & 3.51 & 7 & 0.58 & 5 & 0.33 & 31 & 4.48 & 24.5 & 3.80 & 302.6 & 60.88 \\
\hline 30 & $\begin{array}{l}\text { Sel 98TH1 } \\
744\end{array}$ & ICARDA & $\begin{array}{l}\text { ILC482 } \times \\
\text { NEWC } 36\end{array}$ & Desi & mixed & 3 & 0.33 & 172 & 4.18 & 220 & 2.19 & 7 & 0.58 & 3 & 0.33 & 35 & 2.60 & 30.1 & 1.65 & 1234.4 & 331.10 \\
\hline 31 & $\begin{array}{l}\text { Sel 98TH1 } \\
1518\end{array}$ & ICARDA & not traced & Desi & yellow & 4 & 0.00 & 173 & 4.04 & 220 & 2.00 & 7 & 0.33 & 3 & 0.33 & 36 & 5.81 & 26.4 & 2.42 & 1130.6 & 301.70 \\
\hline 32 & Azad & Iran & $\begin{array}{l}\text { FLIP85-122C/ } \\
\text { FLIP82-150C// } \\
\text { FLIP 86-77C }\end{array}$ & Kabuli & mixed & 3 & 0.33 & 176 & 4.04 & 224 & 1.76 & 2 & 0.33 & 5 & 0.33 & 53 & 2.03 & 29.7 & 0.24 & 2480.8 & 407.61 \\
\hline 33 & $\begin{array}{l}\text { Flip 93- } \\
\text { 261C }\end{array}$ & ICARDA & $\begin{array}{c}\text { X90TH566/ } \\
\text { FLIP87-69C × } \\
\text { LWC219 }\end{array}$ & Kabuli & mixed & 3 & 0.33 & 175 & 4.67 & 222 & 2.67 & 6 & 0.33 & 5 & 0.33 & 53 & 4.58 & 26.5 & 0.21 & 961.4 & 244.24 \\
\hline 34 & $\begin{array}{l}\text { Flip } 98- \\
258 \mathrm{C}\end{array}$ & ICARDA & not traced & Kabuli & aqua & 3 & 0.00 & 184 & 1.53 & 225 & 0.00 & 2 & 0.33 & 6 & 0.00 & 53 & 1.20 & 34.4 & 1.06 & 2464.4 & 735.73 \\
\hline 35 & $\begin{array}{l}\text { Flip 99- } \\
\text { 34C }\end{array}$ & ICARDA & $\begin{array}{c}\text { X96TH8/ } \\
\text { FLIP91-150C × } \\
\text { FLIP91-105C }\end{array}$ & Kabuli & aqua & 3 & 0.00 & 188 & 0.33 & 224 & 1.53 & 2 & 0.33 & 8 & 0.00 & 50 & 3.38 & 33.4 & 0.51 & 2496.6 & 1073.60 \\
\hline 36 & $\begin{array}{c}\text { Flip 99- } \\
\text { 66C }\end{array}$ & ICARDA & $\begin{array}{c}\text { X96TH61/(F91- } \\
\text { 159XILC1278) } \\
\times \text { F91-149C }\end{array}$ & Kabuli & mixed & 3 & 0.00 & 174 & 1.33 & 224 & 0.58 & 2 & 0.00 & 4 & 0.00 & 42 & 2.40 & 32.4 & 0.10 & 966.0 & 209.61 \\
\hline 37 & $\begin{array}{l}\text { Flip 01- } \\
\text { 06C }\end{array}$ & ICARDA & $\begin{array}{c}\text { X98TH23-1- } \\
\text { BH-20/ILC1799 } \\
\times \text { FLIP92-148 }\end{array}$ & Kabuli & aqua & 4 & 0.33 & 178 & 0.67 & 224 & 0.58 & 2 & 0.33 & 8 & 0.00 & 58 & 4.91 & 28.3 & 1.07 & 1987.6 & 563.67 \\
\hline 38 & $\begin{array}{l}\text { Flip 03- } \\
\text { 46C }\end{array}$ & ICARDA & $\begin{array}{c}\text { X99TH 13/ } \\
\text { FLIP93-62C × } \\
\text { FLIP93-50C }\end{array}$ & Kabuli & aqua & 3 & 0.00 & 188 & 1.33 & 226 & 1.76 & 2 & 0.33 & 8 & 0.00 & 65 & 4.48 & 25.6 & 0.00 & 797.4 & 179.66 \\
\hline 39 & $\begin{array}{l}\text { Flip 03- } \\
112 \mathrm{C}\end{array}$ & ICARDA & $\begin{array}{l}\text { X00TH51/ } \\
\text { FLIP 98-52C × } \\
\text { FLIP 98-47C }\end{array}$ & Kabuli & aqua & 3 & 0.00 & 178 & 1.76 & 224 & 0.58 & 2 & 0.33 & 5 & 0.00 & 54 & 1.00 & 29.1 & 0.00 & 893.6 & 227.56 \\
\hline 40 & $\begin{array}{c}\times 2001 \mathrm{TH} \\
45\end{array}$ & ICARDA & $\begin{array}{c}\text { S } 99093 \times \\
\text { S } 98006\end{array}$ & Kabuli & aqua & 3 & 0.00 & 178 & 0.00 & 228 & 0.33 & 6 & 0.00 & 5 & 0.00 & 48 & 0.33 & 35.6 & 0.91 & 1084.0 & 278.19 \\
\hline 41 & $\begin{array}{c}\times 2003 \mathrm{TH} \\
21\end{array}$ & ICARDA & $\begin{array}{c}(\mathrm{S} 00791 \times \\
\text { FLIP 98-23C }) \times \\
\text { ICCV2 }\end{array}$ & Kabuli & aqua & 3 & 0.00 & 172 & 4.67 & 225 & 0.58 & 2 & 1.33 & 8 & 0.00 & 62 & 3.18 & 32.7 & 0.43 & 3086.2 & 938.44 \\
\hline 42 & $\begin{array}{c}\times 2003 \mathrm{TH} \\
164\end{array}$ & ICARDA & $\begin{array}{c}\text { FLIP 98-50C } \times \\
\text { FLIP 97-90C }\end{array}$ & Kabuli & mixed & 3 & 0.00 & 184 & 0.00 & 225 & 0.00 & 2 & 1.33 & 7 & 0.00 & 60 & 2.40 & 30.4 & 0.61 & 846.0 & 195.44 \\
\hline 43 & $\begin{array}{l}\text { C. oxyodon } \\
\text { L.- } 4^{\mathrm{a}}\end{array}$ & Iran & C. oxyodon L. & wild & green & 1 & 0.00 & 169 & 0.33 & 220 & 0.00 & 2 & 0.00 & 2 & 0.00 & 76 & 1.73 & - & & - & \\
\hline 44 & $\begin{array}{l}\text { C. oxyodon } \\
\text { L.-9a }\end{array}$ & Iran & C. oxyodon L. & wild & green & 1 & 0.00 & 169 & 0.33 & 220 & 0.00 & 2 & 0.00 & 2 & & 78 & 1.86 & - & & - & \\
\hline
\end{tabular}

NS - number of stems, DF - days from the first effective raining after sowing to 50\% flowering, DM - days from the first effective raining after sowing to $90 \%$ maturity, SABD - susceptibility to ascochyta blight disease, CT - cold tolerance, PH - plant height, $100 \mathrm{SW}$ - 100-seed weight, YLD - seed yield; ${ }^{\mathrm{a}}$ - accession number, ${ }^{\mathrm{b}}$ - see Figure 3 
Molecular experiments. Genomic DNA was extracted from the young leaf tissues of 2-week-old seedlings using the modified method described by Dellaporta et al. (1983). Nineteen polymorphic SSRs were used for genotyping, from the available composite collections of International Crops Research Institute for the Semi-Arid-Tropics (ICRISAT) and International Center for Agricultural Research in the Dry Areas (ICARDA) (Varshney et al., 2007). Among them, 12 trinucleotide repeat motifs markers of $113-496 \mathrm{bp}$ allele size with annealing temperature of $56-59^{\circ} \mathrm{C}$ (Winter et al., 1999), and 4 di-nucleotide repeat motifs markers of 201-464 bp allele size with annealing temperature of $56^{\circ} \mathrm{C}$ (Sethy et al., 2003) showed polymorphism. The selected markers used in the current study were single locus, co-dominant microsatellite markers whose usefulness in chickpea has already been reported (Udupa et al., 1999; Pandian et al., 2000; Cingilli et al., 2005; Varshney et al., 2007). Polymerase chain reaction (PCR) were performed in a volume of $20 \mu \mathrm{l}$ containing $2.5 \mu \mathrm{M}$ of each SSR primer, $0.4 \mathrm{U}$ of Taq DNA polymerase ("Life Technologies", USA), $100 \mu \mathrm{M}$ of each dNTP ("Promega", France), $2 \mu \mathrm{l}(10 \times)$ PCR buffer, $2 \mathrm{mM} \mathrm{MgCl}_{2}$ ("Promega", France), $\mathrm{ddH}_{2} \mathrm{O}$ and $25 \mathrm{ng}$ template DNA, using a 96well "Eppendorf Mastercycler Gradient" (Type 5331, Eppendorf AG, Germany). Amplification was carried for 35 cycles, each consisting of a denaturation step at $94^{\circ} \mathrm{C}$ for $1 \mathrm{~min}$, annealing at $54-59^{\circ} \mathrm{C}$ (depending on the primers sequence) for $1 \mathrm{~min}$ and an extension step at $72^{\circ} \mathrm{C}$ for 1.5 min. An initial denaturation step at $94^{\circ} \mathrm{C}$ for $3 \mathrm{~min}$, and a final extension step of $10 \mathrm{~min}$ at $72^{\circ} \mathrm{C}$ were also included. The reaction products were mixed with an equal volume of formamide dye $(98 \%$ formamide, $10 \mathrm{mM}$ EDTA (ethylenediaminetetraacetic acid), bromophenol blue and xylene cyanol) and resolved in $3 \%$ agarose gel $(0.5 \times \mathrm{TBE}$ buffer (tris-borate-EDTA)), stained with ethidium bromide (1 $\mu \mathrm{g} \mathrm{ml} \mathrm{m}^{-1}$ ) and photographed under UV (ultraviolet) light. Compared to polyacryl-amide-gel electrophoresis, or automated analysis, agarose-gel electrophoresis is the most-appropriate and safe technology for routine analyses of these types of markers.

Statistical analysis. The SSR data were scored as codominant markers in order to distinguish homozygotes and heterozygotes for each locus. Population structure was analysed using a model-based Bayesian approach in the software package Structure 2.3.4 (Pritchard et al., 2000). Five independent runs were performed, setting the number of sub populations $(\mathrm{K})$ from 1 to 10 , burn in time and Markov chain Monte Carlo (MCMC) replication number both to 100,000 , and a model for admixture and correlated allele frequencies. The $\mathrm{K}$ value was determined by $\operatorname{Ln} \mathrm{P}(\mathrm{D})$ : the $\log$ probability of data in the Structure output and $\Delta \mathrm{K}$ : an ad hoc quantity based on the second order rate of change of the likelihood function with respect to K (Evanno et al., 2005). The appropriate value of $\mathrm{K}$ was determined when the estimate of $\mathrm{Ln}$ $\mathrm{P}(\mathrm{D})$ reached a minimum stable value. Inferred ancestry estimates of individuals (Q-matrix) were derived for the selected subpopulation (Pritchard et al., 2000). Association mapping was performed to analyse markertrait association by structured association approach using ancestry coefficient ( $Q$ values) estimates as covariate in a general linear model (GLM) function using TASSEL 2.1. Multiple testing corrections were performed by adjusting maker probability values for multiple tests run by a permutation test in the TASSEL 2.1 software.

\section{Results}

Analysis of variance revealed significant differences among genotypes for the studied traits (Table 2). The coefficient of variation (CV) ranged from 2.22 to 89.13 . However, for the majority of traits the values were less than $20 \%$ (Table 2 ).

Table 2. Mean of square and some statistical parameters for 44 genotypes of chickpea comprising cultigen, landraces, internationally developed improved lines and wild relatives

\begin{tabular}{ccccccccc}
\hline $\begin{array}{c}\text { Source of } \\
\text { variation }\end{array}$ & NS & $\begin{array}{c}\text { DF } \\
\text { (days) }\end{array}$ & $\begin{array}{c}\text { DM } \\
\text { (days) }\end{array}$ & CT & SABD & $\begin{array}{c}\text { PH } \\
\mathrm{cm}\end{array}$ & $\begin{array}{c}100 \mathrm{SW} \\
\mathrm{g}\end{array}$ & $\begin{array}{c}\text { YLD } \\
\mathrm{kg} \mathrm{ha}^{-1}\end{array}$ \\
\hline Block & $1.1031^{* *}$ & $626.58^{* *}$ & $267.96^{* *}$ & $2.06^{* *}$ & $5.58 \mathrm{~ns}$ & $708.93^{* *}$ & $95.82^{* *}$ & $12057874.70^{* *}$ \\
Genotype & $1.23^{* *}$ & $42.38^{* *}$ & $31.89 \mathrm{~ns}$ & $1.67^{* *}$ & $6.39^{* *}$ & $364.27^{* *}$ & $77.32^{* *}$ & $661609.50^{* *}$ \\
Mean & 3.38 & 179.80 & 224.60 & 1.87 & 3.88 & 40.57 & 26.79 & 637.97 \\
CV & 19.58 & 2.48 & 2.22 & 19.75 & 39.09 & 15.60 & 11.92 & 89.13 \\
Max & 5.00 & 189.30 & 229.00 & 6.00 & 6.70 & 57.00 & 35.70 & 1663.30 \\
Min & 2.00 & 173.60 & 207.00 & 1.30 & 1.30 & 22.30 & 15.00 & 71.00 \\
SE & \pm 0.07 & \pm 0.54 & \pm 0.49 & \pm 0.07 & \pm 0.17 & \pm 1.11 & \pm 0.51 & \pm 70.26 \\
\hline
\end{tabular}

NS - number of stems, DF - days from the first effective raining after sowing to $50 \%$ flowering, DM - days from the first effective raining after sowing to $90 \%$ maturity, $\mathrm{CT}$ - cold tolerance, SABD - susceptibility to ascochyta blight disease, $\mathrm{PH}$ - plant height, $100 \mathrm{SW}$ - 100-seed weight, YLD - seed yield; CV - coefficient of variation, SE - standard error

Mean data revealed high range for most of the studied traits (Table 1). Number of stems per plant ranged from 1 to 7 , days from the first effective raining after sowing to $50 \%$ flowering - from 168 to 188 days, SABD - from 2 (partial resistance) to 8 (highly susceptible), cold tolerance - from 2 (tolerant) to 9 (highly susceptible), plant height - from 16 to $78 \mathrm{~cm}, 100$-seed weight - from
12 to $35.8 \mathrm{~g}$, seed yield - from 31.8 to $4190.4 \mathrm{~kg} \mathrm{ha}^{-1}$ (Table 1).

Genetic diversity among 44 chickpea genotypes was assessed by using 19 microsatellite (SSR) loci (Fig. 1). Three loci were dropped due to missing data $(>5 \%)$. A total number of 100 alleles were detected by 16 SSR primers with an average of 6.25 alleles per locus. 
Detailed information on the levels of genetic diversity of genotypes is available elsewhere (Saeed et al., 2011). Analysis of population structure distinguished 6 subspecific populations (Fig. 1).

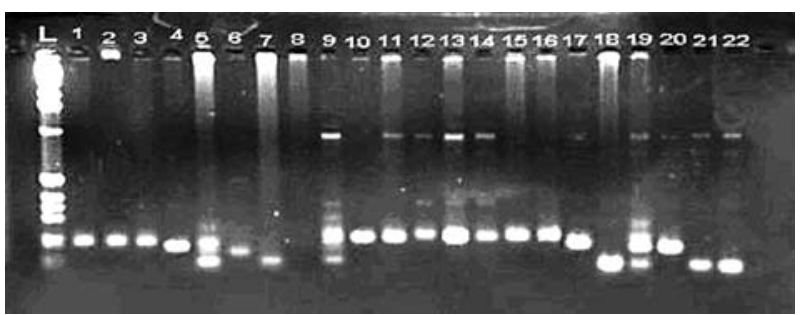

Figure 1. Polymorphism detected by simple sequence repeat (SSR) primer Ta $135 ; \mathrm{L}-1 \mathrm{~kb}$ molecular ladder (genotype codes see in Table 1)

The six subgroups matched rather to the six germplasm groups, landraces, wild relatives, improved lines (Sel 96TH: Desi type), improved lines (Sel 96TH: Kabuli type), improved lines (Flip ) (Figs 2 and 3). Of all genotypes, $70.46 \%$ were assigned into the corresponding subgroups, and the remaining ones were categorized into the mixed subgroups based on their $\mathrm{Q}$ values (Table 1 and Fig. 3).

By applying GLM procedure and multiple testing corrections in the TASSEL 2.1 software, 3, 2, 1, 3 , and 1 SSR loci were identified to be associated with genes controlling stem number, days from first effective raining after sowing to $90 \%$ maturity, 100 -seed weight,
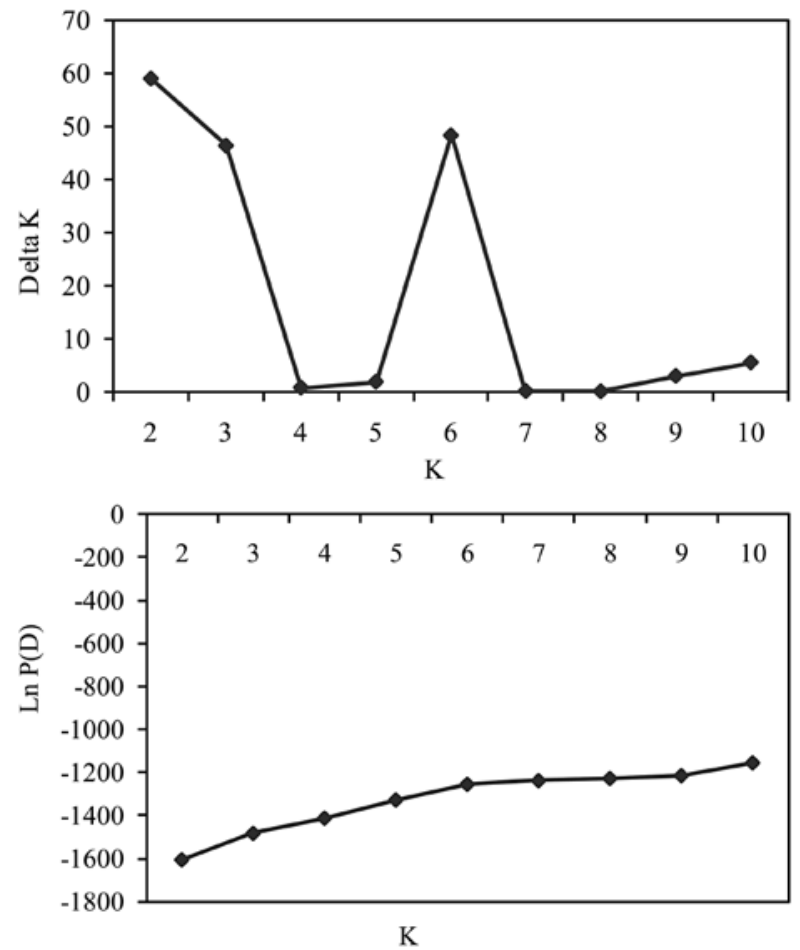

Note. $\mathrm{Ln} \mathrm{P}(\mathrm{D})$ is the log likelihood of the observed genotype distribution in $\mathrm{k}$ clusters and is an output by Structure simulation, $\Delta \mathrm{K}$ - an ad hoc quantity based on the second order rate of change of the likelihood function with respect to $K$.

Figure 2. Bilateral charts to determine the optimal number of K identified by Structure program
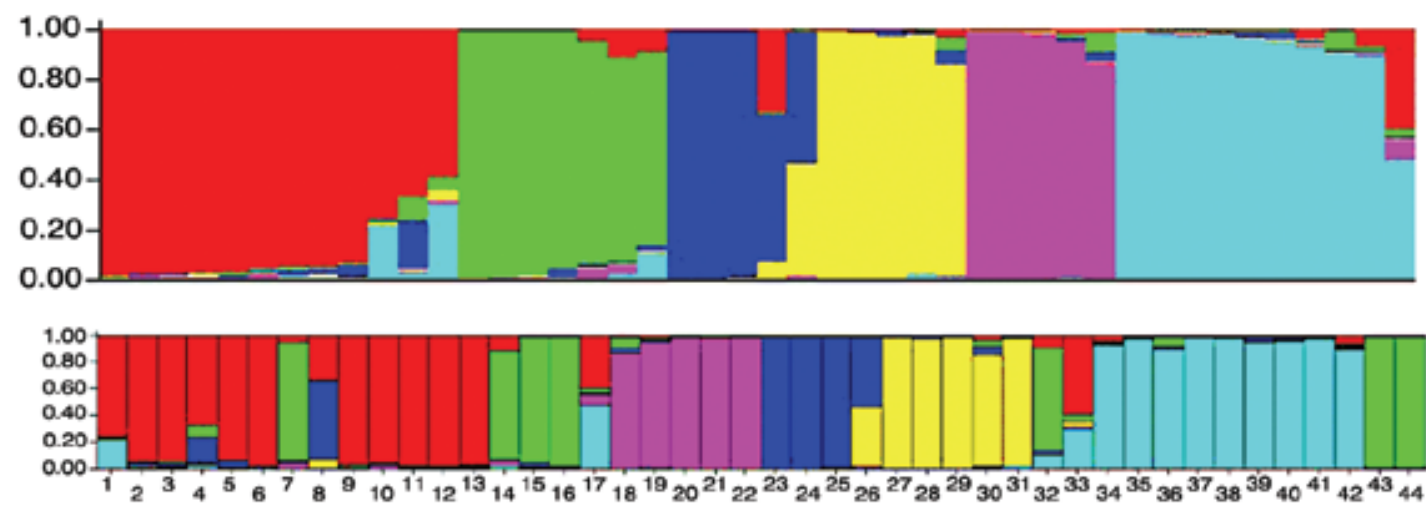

Notes. Numbers on the y-axis indicate the membership coefficient. The colour of the bar indicates the six groups identified through the Structure program (red - landraces, green - wild relatives, blue and yellow - improved lines (Sel 96TH1: Desi type), pink

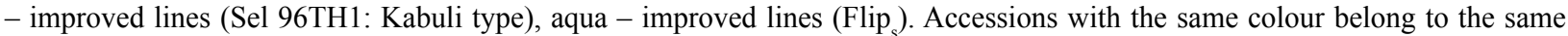
subgroup.

Figure 3. Genetic relatedness of 44 genotypes of chickpea comprising cultigen, landraces, internationally developed improved lines and wild relatives with 16 simple sequence repeats (SSRs) as analysed by the Structure program

plant height, and cold tolerance, respectively (Table 3 ). None of identified markers for yield and partial resistance to ascochyta blight disease passed the stringent bar of statistical significance, and all $P$-values were greater (Table 3$)$. The phenotypic variance explained by loci $\left(R^{2}\right)$ ranged from $8 \%$ to $75 \%$ (Table 3 ).

Among the identified SSR loci, two SSR loci were common whereas the others were specific. The
SSR locus TS84 was common for the majority of traits including stem number, days from first effective raining after sowing to $90 \%$ maturity, 100 -seed weight, plant height, and cold tolerance. The phenotypic variance explained by this marker $\left(R^{2}\right)$ in different traits ranged from $31 \%$ to $68 \%$. The locus Ta142 was common for stem number and plant height. 
Table 3. Single sequence repeats (SSR) loci identified for studied agro-morphological traits in chickpea using association mapping by general linear model (GLM) procedure

\begin{tabular}{cccccc}
\hline Trait & Locus & $F$-marker $^{\mathrm{a}}$ & $P$-marker & $P$-adj marker & $R^{2}$ \\
\hline NS & Ta118 & 3.3918 & 0.009 & $0.064 \mathrm{~ns}$ & 0.4809 \\
NS & Ta135 & 6.3815 & 0.000 & $0.001^{*}$ & 0.7494 \\
NS & Ta142 & 9.0165 & 0.000 & $0.251 \mathrm{~ns}$ & 0.5652 \\
NS & Tr43 & 2.8113 & 0.019 & $0.001^{*}$ & 0.5002 \\
NS & TS84 & 11.6027 & 0.000 & $0.309 \mathrm{~ns}$ & 0.68 \\
DM & NCP6 & 2.8917 & 0.017 & $0.028^{*}$ & 0.0822 \\
DM & NCP7 & 7.5115 & 0.010 & $0.443 \mathrm{~ns}$ & 0.2921 \\
DM & Ta135 & 2.6583 & 0.028 & $0.595 \mathrm{~ns}$ & 0.2334 \\
DM & Tr43 & 2.5068 & 0.032 & $0.026^{*}$ & 0.3132 \\
DM & TS84 & 4.2344 & 0.003 & $0.444 \mathrm{~ns}$ & 0.0652 \\
SABD & NCP19 & 3.6472 & 0.038 & $0.401 \mathrm{~ns}$ & 0.2566 \\
CT & Taa58 & 2.8947 & 0.029 & $0.081 \mathrm{~ns}$ & 0.3292 \\
CT & Tr43 & 3.3142 & 0.007 & $0.010^{*}$ & 0.3719 \\
CT & TS84 & 4.3087 & 0.002 & $0.732 \mathrm{~ns}$ & 0.3114 \\
PH & Ta118 & 2.3423 & 0.048 & $0.015^{*}$ & 0.3397 \\
PH & Ta142 & 4.7652 & 0.003 & $0.007^{*}$ & 0.4345 \\
PH & Tr43 & 4.2329 & 0.002 & $0.036^{*}$ & 0.4234 \\
PH & TS84 & 4.1408 & 0.003 & $0.692 \mathrm{~ns}$ & 0.289 \\
100 SW & NCP6 & 2.3848 & 0.041 & $0.543 \mathrm{~ns}$ & 0.1618 \\
100 SW & Tr29 & 2.7239 & 0.041 & $0.074 \mathrm{~ns}$ & 0.2748 \\
100 SW & Tr43 & 3.3994 & 0.007 & $0.001^{*}$ & 0.4126 \\
100 SW & TS84 & 6.1875 & 0.000 & $0.292 \mathrm{~ns}$ & 0.4951 \\
YLD & NCP6 & 2.8295 & 0.019 & $0.945 \mathrm{~ns}$ & 0.1952 \\
YLD & Tr7 & 2.6011 & 0.091 & $0.134 \mathrm{~ns}$ & 0.5262 \\
YLD & TS84 & 3.1343 & 0.014 & $90 \% \mathrm{~ns}$ & \\
\hline
\end{tabular}

Notes. NS - number of stems, DM - days from the first effective raining after sowing to $90 \%$ maturity, SABD - susceptibility to ascochyta blight disease, CT - cold tolerance, $\mathrm{PH}$ - plant height, $100 \mathrm{SW}-100$-seed weight, YLD - seed yield; ${ }^{\text {a, b }}-F$-statistics and $p$-values for the requested $F$-tests, ${ }^{\mathrm{c}}$ - the marker $p$-value adjusted for multiple tests (Ge et al., 2003); $R^{2}-$ the portion of total variation explained by the marker but not by the other terms in the model.

\section{Discussion}

High genetic variability was observed among the chickpea genotypes for the studied agro-morphological traits. High genetic variability observed among advanced breeding lines indicates the efforts underway in ICARDA to widen the genetic base of chickpea for various traits. A higher variation for a character in the breeding materials correlates with a greater ability for its improvement through selection. The CV for the majority of traits was less than $20 \%$. In general, CV value higher than $20 \%$ is considered to be high; however, it may be possible to ignore the high $\mathrm{CV}$ values when $\mathrm{F}$ test is significant and this item is found in several published research works (Okwuagwu et al., 2008; Kandic et al., 2009). The inconsistent $\mathrm{CV}$ values reported in many studies might be due to physio-genetic characteristics and degree of compatibility of the plant material, low number of sampled individuals per genotype in plot, low number of replications per genotype and/or variable environments in which the trial was carried out (Okwuagwu et al., 2008).

In this study wild Cicer oxyodon accessions showed cold tolerance and high partial resistance against ascochyta blight disease. These results are in agreement with the findings of Toker (2005) who observed high cold tolerance in wild relatives of Cicer such as $C$. reticulatum. Wild relatives of Cicer species (Singh et al., 2008) and landraces are a promising source of genes for stress tolerance. Inter-specific hybridization has opened up the possibility of transferring genes from wild to cultivated species (Singh, Ocampo, 1997). The present study used 16 pairs of SSR primers and subdivided the association population into six subpopulations using the modelbased cluster method. Data from our genetic diversity (Saeed et al., 2011) and population structure analysis revealed that this association population showed a diverse genetic variation and, therefore, could be used for the association analysis. Among factors influencing marker-traits association, the admixture of populations is very important (Zhang et al., 2012). Many models were used to minimize the false-positive of association analysis produced from the admixture of populations. It has been showed that a $\mathrm{K}$ matrix incorporated into the GLM was sufficient to minimize false-positive associations (Zhang et al., 2012). Using GLM method several loci associated with genes controlling traits were identified. A great challenge in association mapping via GLM method is instability of the experiment-wise Type I error. When multiple hypothesis tests are performed, the experiment-wise Type I error is quickly increased, which results in false positives. To reduce the probability of false positives, multiple testing corrections were used for controlling experiment-wise Type I error. Multiple corrections test was used to control experiment-wise Type I error in several association mapping studies such as Vanniarajan et al. (2012). Cho et al. (2002) by constructing an intraspecific genetic linkage map on $\mathrm{F}_{10}$ population via 55 sequence-tagged microsatellite 
sites (STMS), 20 random amplified polymorphic DNAs (RAPDs), 3 intersimple sequence repeats (ISSR) and 2 phenotypic markers, determined the map positions of genes controlling days to $50 \%$ flowering, double podding, seed number per plant and 100-seed weight traits in chickpea. Sharma et al. (2004) identified the STMS and sequence-tagged site (STS) markers closely linked to resistance gene against Fusarium oxysporum $\mathrm{f}$. sp. ciceris race 3 in chickpea by QTL mapping.

Identification of molecular markers using unrelated germplasm collections instead of utilizing materials derived from planned crosses such as F2, RIL, doubled haploid (DH) populations has increased in most of the laboratories in recent years (Roy et al., 2006; HajiAllahverdipoor et al., 2011; Vanniarajan et al., 2012; Zhang et al., 2012). This approach could identify markers with pleiotropic effects indicating that population wide analysis served as an effective tool in deciphering marker-trait associations. Detection of molecular markers influencing various traits could increase the efficiency of marker-assisted selection and enhance genetic progress.

\section{Conclusion}

The present study offers an approach for identifying a set of deoxyribonucleic acid (DNA) markers projecting significant association with genes controlling agro-morphological traits in chickpea. Using general linear model (GLM) and applying multiple testing corrections, several simple sequence repeat (SSR) markers were identified. Some identified markers associate with more than one trait. Detection of molecular markers associated with genes controlling different traits could increase the efficiency of marker-assisted selection (MAS) in chickpea breeding programs.

\section{Acknowledgments}

The authors are grateful to the Institute of Biotechnology, Urmia University, Iran for the scientific support of this work.

Received 22012013

Accepted 13062013

\section{References}

Ahmad F., Gaur P. M., Croser J. 2005. Chickpea (Cicer arietinum $\mathrm{L}$.). Genetic resources, chromosome engineering and crop improvement. Singh R. J., Jauhar P. P. (eds). Grain Legumes, 1: 187-217

Chaturvedi S. K., Nadarajan N. 2010. Genetic enhancement for grain yield in chickpea- accomplishments and resetting research agenda. Electronic Journal of Plant Breeding, 1 (4): 611-615

Cho S., Kumar J., Schultz J. L., Anupama K., Tefera F., Muehlbauer F. J. 2002. Mapping genes for double podding and other morphological traits in chickpea. Euphytica, 128: 285-292 http://dx.doi.org/10.1023/A:1020872009306

Cingilli H., Altinkut A., Akcin A. 2005. The use of microsatellite markers in the annual and perennial Cicer species growing in Turkey. Biologia: section Botany, 60 (1): 93-98

Darvishzadeh R., Poormohammad Kiani S., Huguet T., Sarrafi A. 2008. Genetic variation and identification of molecular marker associated with partial resistance to Phoma macdonaldii in gamma-irradiation-induced mutants of sunflower. Canadian Journal of Plant Pathology, 30: 106-114 http://dx.doi.org/10.1080/07060660809507501

Dellaporta S. L., Wood J., Hicks J. B. 1983. A plant DNA mini preparation. Plant Molecular Biology Reporter, 1: 19-21 http://dx.doi.org/10.1007/BF02712670

Evanno G., Regnaut S. Goudet J. 2005. Detecting the number of clusters of individuals using the software STRUCTURE: a simulation study. Molecular Ecology, 14: 2611-2620 http://dx.doi.org/10.1111/j.1365-294X.2005.02553.x

Gaur R., Sethy N. K., Choudhary S., Shokeen B., Gupta V., Bhatia S. 2011. Advancing the STMS genomic resources for defining new locations on the intraspecific genetic linkage map of chickpea (Cicer arietinum L.). BMC Genomics, 12: 117 http://dx.doi.org/10.1186/1471-2164-12-117

Ge Y., Dudoit S., Speed T. P. 2003. Resampling-based multiple testing for microarray data analysis. Test, 12: 1-77

Haji-Allahverdipoor K., Bahramnejad B., Amini J. 2011. Selection of molecular markers associated with resistance to Fusarium wilt disease in chickpea (Cicer arietinum L.) using multivariate statistical techniques. Australian Journal of Crop Science, 5 (13): 1801-1809

Jain D., Chattopadhyay D. 2010. Analysis of gene expression in response to water deficit of chickpea (Cicer arietinum L.) varieties differing in drought tolerance. BMC Plant Biology, 10: 24 http://dx.doi.org/10.1186/1471-2229-10-24

Kandic V., Dodig D., Jovic M., Nikolic B., Prodanovic S. 2009. The importance of physiological traits in wheat breeding under irrigation and drought stress. Genetika, 41: 11-20 http://dx.doi.org/10.2298/GENSR0901011K

Kanouni H., Taleei A., Okhovat M. 2010. Ascochyta blight (Ascochyta rabiei (Pass.) Lab.) of chickpea (Cicer arietinum L.): Breeding strategies. International Journal of Plant Breeding and Genetics, 5: 1-22

Mittler R., Blumwald E. 2010. Genetic engineering for modern agriculture: challenges and perspectives. Annual Review of Plant Biology, 2 (61): 443-462 http://dx.doi.org/10.1146/annurev-arplant-042809-112116

Okwuagwu C. O., Okoye M. N., Okolo E. C., Ataga C. D., Uguru M. I. 2008. Genetic variability of fresh fruit bunch yield in Deli/dura $\times$ tenera breeding populations of oil palm (Elaeis guineensis Jacq.) in Nigeria. Journal of Tropical Agriculture, 46: 52-57

Pandian A., Ford R., Taylor P. W. J. 2000. Transferability of sequence tagged microsatellite site (STMS) primers across four major pulses. Plant Molecular Biology Reporter, 18: 395a-395h http://dx.doi.org/10.1007/BF028250669

Pritchard J. K., Stephens M., Donnelly P. 2000. Inference of population structure using multilocus genotype data. Genetics, 155: 945-959

Pritchard J. K., Donnelly P. 2001. Case-control studies of association in structured or admixed populations. Theoretical Population Biology, 60: 227-237 http://dx.doi.org/10.1006/tpbi.2001.1543

Rajesh P. N., Muehlbauer F. J. 2008. Discovery and detection of single nucleotide polymorphism (SNP) in coding and genomic sequences in chickpea (Cicer arietinum L.). Euphytica, 162: 291-300 http://dx.doi.org/10.1007/s10681-008-9675-8

Roy J. K., Bandopadhyay R., Rustgi S., Balyan H. S., Gupta P. K. 2006. Association analysis of agronomically important traits using SSR, SAMPL and AFLP markers in bread wheat. Current Science, 90: 683-689

Saeed A., Darvishzadeh R., Hovsepyan H., Asatryan A. 2010. Tolerance to freezing stress in Cicer accessions under controlled and field conditions. African Journal of Biotechnology, 9 (18): 2618-2626

Saeed A., Hovsepyan H., Darvishzadeh R., Imtiaz M., Panguluri S. K., Nazaryan R. 2011. Genetic diversity in Iranian accessions, improved lines of chickpea (Cicer 
arietinum L.) and their wild relatives by using simple sequence repeats. Plant Molecular Biology Reporter, 29 (4): $848-858$

http://dx.doi.org/10.1007/s11105-011-0294-5

Sethy N. K., Shokeen B., Bhatia S. 2003. Isolation and characterization of sequence-tagged microsatellite sites markers in chickpea (Cicer arietinum L.). Molecular Ecology Notes, 3:428-430 http://dx.doi.org/10.1046/j.1471-8286.2003.00472.x

Sharma K. D., Winter P., Kahl G., Muehlbauer, F. J. 2004. Molecular mapping of Fusarium oxysporum f. sp. ciceris race 3 resistance gene in chickpea. Theoretical and Applied Genetics, 108: 1243-1248 http://dx.doi.org/10.1007/s00122-003-1561-0

Singh K. B., Ocampo B. 1997. Exploitation of wild Cicer species for yield improvement in chickpea. Theoretical and Applied Genetics, 95: 418-423 http://dx.doi.org/10.1007/s001220050578

Singh R., Sharma P., Varshney R. K., Sharma S. K., Singh N. K. 2008. Chickpea improvement: role of wild species and genetic markers. Biotechnology and Genetic Engineering Reviews, 25: 267-314 http://dx.doi.org/10.5661/bger-25-267

Toker C. 2005. Preliminary screening and selection for cold tolerance in annual wild Cicer species. Genetic Resources and Crop Evolution, 52: 1-5 http://dx.doi.org/10.1007/s10722-005-1743-5

Udupa S. M., Robertson L. D., Weigand F., Baum M., Kahl G. 1999. Allelic variation at (TAA)n microsatellite loci in a world collection of chickpea (Cicer arietinum L.) germplasm. Molecular Genetics and Genomics, 26: 354-63

Vanniarajan C., Vinod K. K., Pereira A. 2012. Molecular evaluation of genetic diversity and association studies in rice (Orvza sativa L.). Journal of Genetics, 91: 9-19 http://dx.doi.org/10.1007/s12041-012-0146-6

Varshney R. K., Hoisington D. A., Upadhyaya H. D., Gaur P. M., Nigam S. N., Saxena K. B., Vadez V., Sethy N. K., Bhatia S., Aruna R., Gowda M. V. C., Singh N. K. 2007. Molecular genetics and breeding of grain legume crops for the semiarid tropics. Genomics assisted crop improvement. Genomics applications. Varshney R. K. et al. (eds). Crops. II, p. 207-242

Vijayan K., Srivatsava P. P., Nair C.V., Awasthi A. K., Tikader A., Sreenivasa B., Urs S. R. 2005. Molecular characterization and identification of markers associated with yield traits in mulberry using ISSR markers. Plant Breeding. 125: 298301 http://dx.doi.org/10.1111/j.1439-0523.2006.01212.x

Winter P., Pfaff T., Udupa S. M., Hüttel B., Sharma P. C., Sahi S., Arreguin-Espinoza R., Weigand F., Muehlbauer F. J., Kahl G. 1999. Characterisation and mapping of sequence-tagged microsatellite sites in the chickpea (Cicer arietinum L.) genome. Molecular Genetics and Genomics, 262: 90-101 http://dx.doi.org/10.1007/s004380051063

Zhang Q., Wu C., Ren F., Li Y., Zhang C. 2012. Association analysis of important agronomical traits of maize inbred lines with SSRs. Australian Journal of Crop Science, 6 (6): $1131-1138$

\title{
Sẻjamojo avinžirnio (Cicer arietinum L.) paprastụjų pasikartojančių sekų žymeklių ir agromorfologinių požymių asociacijų analizè
}

\author{
A. Saeed ${ }^{1}$, R. Darvishzadeh ${ }^{2,3}$, A. Basirnia ${ }^{2}$ \\ ${ }^{1}$ Vakarų Azerbaidžano žemės ūkio ir gamtos išteklių tyrimų centras, Iranas \\ ${ }^{2}$ Urmia universiteto Augalu selekcijos ir biotechnologijos fakultetas, Iranas \\ ${ }^{3}$ Urmia universiteto Biotechnologijos institutas, Iranas
}

\section{Santrauka}

Sejamasis avinžirnis (Cicer arietinum L.), šaltojo sezono pupinis augalas, besivystančiose šalyse yra svarbus ir pigus baltymų bei energijos šaltinis, dirvožemị papildantis maisto medžiagomis. Siekiant identifikuoti paprastujjų pasikartojančių sekų (PPS) žymeklius, susijusius su sėjamojo žirnio agromorfologiniais požymiais, buvo tirta 44 genotipų kolekcija, sudaryta iš kultigeno, vietinių veislių, pagerintų selekcinių linijų ir laukinių ekotipų. Tyrimas atliktas Urmia tyrimų stotyje lietinimo sąlygomis, taikant atsitiktine tvarka išdèstyto viso bloko schemą, trimis pakartojimais. Nustatyta didelè sẻjamojo avinžirnio agromorfologinių požymių genetinè ịvairovè. Genotipu genetinè įvairovė nustatyta molekulinio tyrimo metu, naudojant PPS žymeklius. Šešiolikoje PPS lokusų nustatyta 100 alelių, vidutiniškai 6,25 alelio viename lokuse. Taikant bendrajị linijini modeli ir kartotines tyrimo korekcijas identifikuota 10 PPS lokusų, susijusių su genais, kontroliuojančiais tirtus agromorfologinius požymius. Kai kurie identifikuoti žymekliai yra susiję su daugiau nei vienu požymiu. Identifikuoti žymekliai gali būti naudingi vykdant žymekliais pagrịstą atranką avinžirnio selekcinèse programose.

Reikšminiai žodžiai: asociacijų analizè, Cicer arietinum, genetinè ịvairovė, molekuliniai žymekliai. 